\title{
Determinación de carbono orgánico en materias primas cerámicas en presencia de siderita, $\mathrm{FeCO}_{3}$
}

\author{
M. F. GAZULLA; C. M. GÓMEZ; M. RODRIGO; M. ORDUÑA \\ Instituto de Tecnología Cerámica. \\ Asociación de Investigación de las Industrias Cerámicas. \\ Universitat Jaume I. Castellón. España.
}

\begin{abstract}
La materia orgánica es una de las impurezas presentes en las materias primas utilizadas en la fabricación de pavimentos y revestimientos cerámicos. Durante el proceso de cocción, el carbono orgánico (Corgánico) puede producir la aparición de un núcleo negro conocido como "corazón negro", que puede causar defectos en el producto final.

Las materias primas cerámicas contienen Corgánico a nivel de $\mathrm{mg} \cdot \mathrm{kg}^{-1}$ (procedente de la materia orgánica) y carbono inorgánico (Cinorgánico) a nivel de porcentaje (procedente de siderita $\left(\mathrm{FeCO}_{3}\right)$, calcita $\left(\mathrm{CaCO}_{3}\right)$ y dolomita $\left(\mathrm{CaMg}\left(\mathrm{CO}_{3}\right)_{2}\right)$, principalmente).

La presencia de siderita puede interferir en la determinación de Corgánico puesto que su temperatura de descomposición difiere muy poco de la temperatura de determinación del Corgánico, no ocurriendo lo mismo con la calcita y la dolomita, cuyas temperaturas de descomposición son superiores. Existen diversos métodos para la determinación de Corgánico en muestras sólidas, aunque no se han encontrado estudios cuantitativos sobre dicha determinación en presencia de siderita. En este trabajo se han desarrollado dos métodos para determinar Corgánico en materias primas y composiciones cerámicas mediante análisis elemental, en presencia de siderita, que pueden ser utilizados en el control de la aparición de corazón negro en baldosas cerámicas. La metodología desarrollada presenta bajos límites de cuantificación e incertidumbres, siendo un método rápido y preciso.
\end{abstract}

Palabras clave: carbono orgánico, siderita, analizador elemental, materias primas y productos cerámicos.

\section{INTRODUCCIÓN}

En el proceso cerámico, concretamente en la fabricación de pavimento y revestimiento cerámicos, se utilizan arcillas que habitualmente suelen contener materia orgánica como impureza. Durante el proceso de cocción puede aparecer un núcleo oscuro en el interior de la pieza denominado "corazón negro", y está relacionado con la concentración de carbono orgánico presente en las composiciones utilizadas en la preparación de las piezas (1-4), así como por la presencia de óxidos de hierro (5). La aparición de dicho núcleo negro durante la cocción de pavimentos y revestimientos cerámicos viene favorecida por la utilización de ciclos de cocción cortos o la fabricación de piezas de gran formato donde se aumenta la compactación, y con ello disminuye el coeficiente de difusión.

La aparición de este núcleo negro, en la mayoría de los casos, es considerada como un defecto, ya que puede provocar una disminución de la resistencia mecánica del material en el caso de productos vitrificados, hinchamiento de las piezas, deformaciones piroplásticas, alteraciones del tono del soporte y/o esmalte, etc., por lo que se hace necesario encontrar la forma de eliminarlo y/o impedir que se forme, antes de que pueda originar problemas que afecten a la calidad del producto acabado. Por ello, además de una serie de variables del proceso, tales como, la temperatura y el ciclo de cocción, la densidad aparente, el tamaño de las piezas, etc. una variable muy importante a tener en cuenta es la concentración de carbono orgánico que presentan las materias primas utilizadas en la fabricación de las pastas cerámicas.

Las materias primas cerámicas generalmente contienen carbono orgánico a nivel de ppm (el que procede de la materia orgánica y puede provocar la aparición de corazón negro) y carbono inorgánico a nivel de porcentaje (presente en la siderita $\left(\mathrm{FeCO}_{3}\right)$, calcita $\left(\mathrm{CaCO}_{3}\right)$ y dolomita $\left(\mathrm{CaMg}\left(\mathrm{CO}_{3}\right)_{2}\right)$ principalmente).

La presencia de siderita puede generar una importante interferencia en la determinación de carbono orgánico ya que la temperatura de descomposición de ésta difiere muy poco de la temperatura a la cual debe ser sometido un material para analizar el contenido total de carbono orgánico (6-11); no ocurre lo mismo con la calcita y la dolomita, que presentan temperaturas de descomposición superiores. Es la necesidad de analizar carbono orgánico en muy bajas concentraciones en presencia de carbono inorgánico en concentraciones superiores lo que genera la dificultad de hallar métodos lo suficientemente precisos y exactos.

Existen diversos métodos para la determinación de carbono en muestras sólidas, por una parte aquellos métodos 
en los que se realiza la determinación de carbono inorgánico por descomposición con $\mathrm{HCl}$ (12-15), por otra, aquéllos en los que se lleva a cabo la determinación de carbono orgánico mediante tratamiento de la muestra con una mezcla oxidante $\left(\mathrm{K}_{2} \mathrm{Cr}_{2} \mathrm{O}_{7}\right)$ en medio fuertemente ácido (16-19), o mediante oxidación con agua oxigenada (20), siendo métodos lentos y con una incertidumbre elevada. Pero en la mayoría de los casos la determinación se realiza mediante un tratamiento de la muestra a una temperatura elevada (superior a $1000^{\circ} \mathrm{C}$ ) y se determina el carbono total, es decir, la suma de carbono orgánico, carbono inorgánico y carbono elemental (21). En un trabajo reciente se han analizado las diferentes formas de carbono en materiales geológicos (22). En otro trabajo reciente (23) se realiza un estudio sobre la eliminación de siderita para llevar a cabo la determinación de carbono orgánico mediante un análisis por espectrometría de masas, en el cual se necesitan tratamientos ácidos muy prolongados, los resultados hallados presentan elevadas incertidumbres y el método propuesto es complejo.

En este trabajo se han desarrollado dos métodos sencillos y rápidos para la determinación de carbono orgánico en bajas concentraciones en materias primas y pastas cerámicas en presencia de siderita, con objeto de utilizarlo como control en la aparición de corazón negro en piezas cerámicas. Para ello se han estudiado las siguientes variables:

- el tipo de analizador elemental más adecuado para llevar a cabo la determinación

- la temperatura óptima de trabajo para analizar cada forma de carbono (C orgánico y C inorgánico),

- el efecto de la presencia de siderita $\left(\mathrm{FeCO}_{3}\right)$ en la determinación de $\mathrm{C}$ orgánico con un equipo con horno eléctrico,

- la determinación de C orgánico en un equipo con horno de inducción con tratamiento previo de las muestras,

- la relación entre la concentración de C orgánico y la aparición de corazón negro.

Posteriormente, se ha realizado la validación de los métodos desarrollados mediante el uso de materiales de referencia y se ha calculado la incertidumbre de los mismos.

\section{PARTE EXPERIMENTAL}

\subsection{Materiales}

Para la calibración y la validación de las medidas se utilizaron los materiales de referencia: GBW 07401 Soil, GBW 07402 Soil, GBW 07403, GBW 07406 y GBW 07407 suministrados por China National Center for Iron and Steel, Beijing (China) cuya concentración de C orgánico se detalla en la tabla I. Dichos patrones también fueron utilizados para la elección del equipo más adecuado.

Para la optimización de la temperatura de trabajo se utilizó una arcilla con un contenido de C orgánico de $0,037 \%$ con la referencia: ARCILLA T. También se analizaron seis arcillas que se referenciaron con números correlativos desde ARCILLA 1 hasta ARCILLA 6.

Para estudiar la interferencia que genera la siderita en la medida, se prepararon mezclas que contenían las citadas arcillas y siderita en diferentes proporciones. La siderita utilizada para la preparación de dichas mezclas fue el material NCS DC 14038 Siderite suministrado por National Research Center of Certified Reference Standards of the Ministry of Geology and Mineral Resources, Beijing.

TABLA I: MATERIALES DE REFERENCIA UTILIZADOS EN LA CALIBRACIÓN Y VALIDACIÓN DE LAS MEDIDAS

\begin{tabular}{|c|c|}
\hline Material de referencia & Valor certificado $\left(\% \mathbf{C}_{\text {org }}\right)$ \\
\hline GBW 07401 Soil & $1,80 \pm 0,16$ \\
\hline GBW 07402 Soil & $0,49 \pm 0,07$ \\
\hline GBW 07403 Soil & $0,51 \pm 0,03$ \\
\hline GBW 07406 Soil & $0,81 \pm 0,09$ \\
\hline GBW 07407 Soil & $0,64 \pm 0,07$ \\
\hline
\end{tabular}

Para el estudio de la relación entre la concentración de carbono orgánico y la aparición de corazón negro, se utilizaron tres arcillas y tres atomizados con las siguientes referencias: ARCILLAS A, B, y C, y ATOMIZADOS A, B y C. Los atomizados son una mezcla de materias primas (arcillas, arenas silíceas y feldespatos, principalmente) formadas por gránulos más o menos esféricos, huecos en su interior y muy homogéneos, que se utilizan para la preparación de soportes cerámicos.

Todos los materiales fueron molturados en un molino de anillos de carburo de wolframio, ya que se ha comprobado que para asegurar una recuperación del analito próxima al $100 \%$ en la medida de carbono es necesario que los materiales posean un tamaño de partícula inferior a $75 \mu \mathrm{m}$.

\subsection{Equipos}

Los equipos utilizados para la determinación de carbono han sido los analizadores elementales modelo RC-412, modelo CS-200 y modelo TruSpec CHN todos ellos de la firma LECO.

El analizador elemental modelo RC-412 dispone de un horno tubular donde se introduce la muestra (entre $100 \mathrm{mg}$ y 0,5 g dependiendo de la cantidad de carbono en la muestra). Ésta se somete a un proceso de calentamiento donde se produce la combustión y se desprende $\mathrm{CO}_{2}$ y $\mathrm{H}_{2} \mathrm{O}$. Por el tubo circula una corriente de oxígeno que arrastra los gases emitidos en la combustión hasta dos celdas de infrarrojo (IR) donde se analizan selectivamente el $\mathrm{CO}_{2}$ y el $\mathrm{H}_{2} \mathrm{O}$. El equipo permite programar la temperatura de forma que es posible la diferenciación entre los diferentes tipos de carbono presentes en la muestra: C orgánico, $\mathrm{C}$ inorgánico.

El analizador de elemental modelo CS-200 posee un horno de inducción que alcanza temperaturas muy elevadas (alrededor de $2000{ }^{\circ} \mathrm{C}$ ) en el cual se introduce la muestra y se produce la combustión de ésta. La muestra se coloca en un crisol de alúmina (entre $100 \mathrm{mg}$ y $0,5 \mathrm{~g}$ dependiendo de la cantidad de carbono presente en la muestra) donde se mezcla con hierro para favorecer el proceso de inducción para muestras no conductoras, y en el caso de muestras poco reactivas, con wolframio, que actúa como catalizador de la fusión. Por el horno circula una corriente de oxígeno puro, que, junto con el calor generado por inducción, provoca la combustión de la muestra. Durante la combustión se liberan $\mathrm{CO}_{2} \mathrm{y} \mathrm{SO}_{2^{\prime}}$ entre otros gases que son retenidos en trampas, de forma que únicamente estos dos gases alcanzan el sistema de 
detección, que está formado por dos celdas de IR, una para la detección del $\mathrm{CO}_{2}$ y otra para la del $\mathrm{SO}_{2}$.

El analizador elemental modelo TruSpec CHN, también puede trabajar a temperatura variable $\mathrm{y}$, por lo tanto permite determinar, tanto el carbono orgánico, como el inorgánico y el total. Posee un horno donde se introduce la muestra (entre 50 y $200 \mathrm{mg}$ dependiendo de las cantidades de carbono presentes en la muestra), por donde circula una corriente de oxígeno. En el horno se produce la combustión de la muestra y los gases generados son arrastrados por la corriente de oxígeno hasta un recipiente de homogeneización. Una vez homogeneizados los gases en dicho recipiente, son conducidos, por una parte por una corriente de oxígeno hacia las celdas de infrarrojo para la determinación del carbono y del hidrógeno, y por otra parte, por una corriente de helio hacia unaa celda de termoconductividad para la determinación de nitrógeno.

Para la obtención de las probetas utilizadas en el ensayo de formación de corazón negro se utilizó una prensa hidráulica de la firma CASMON y un horno mufla de laboratorio de la firma NANNETTI para la cocción de las mismas.

\subsection{Procedimiento experimental}

A continuación se describen las diferentes variables analizadas en este estudio.

\subsubsection{OPTIMIZACIÓN DE LA TEMPERATURA DE} TRABAJO PARA LA MEDIDA DIRECTA DE C ORGÁNICO EN UN HORNO ELÉCTRICO Y EFECTO DE LA PRESENCIA DE SIDERITA

Se han encontrado numerosos estudios en los que se realiza la determinación de carbono orgánico a diferentes temperaturas. Krom y Berner (1983) (24) desarrollaron un método en el que una muestra era analizada en dos condiciones diferentes: una porción a $1350{ }^{\circ} \mathrm{C}$ sin ningún tratamiento previo, y otra porción a la misma temperatura pero después de un tratamiento a $450{ }^{\circ} \mathrm{C}$ y el carbono orgánico se obtenía por diferencia. Según otros autores (25), el carbono orgánico se oxida completamente a $420{ }^{\circ} \mathrm{C}$ utilizando un tiempo de análisis de 15 minutos, mientras que los carbonatos (calcita y dolomita) se descomponen completamente sometiéndolos a una temperatura de $850{ }^{\circ} \mathrm{C}$ durante 30 minutos. Sin embargo, Kissinger, et. al. (1955) (7) establecieron anteriormente que la siderita se descompone completamente a $500{ }^{\circ} \mathrm{C}$.

Realmente lo importante es establecer a qué temperatura se ha descompuesto completamente el carbono orgánico y no han comenzado a descomponerse los carbonatos, y en un estudio más reciente, Wang y Anderson (1998) (25), muestran que a una temperatura menor de $700{ }^{\circ} \mathrm{C}$ no se consigue una recuperación total del carbono orgánico, en los materiales en los cuales se realizó el estudio, y proponen el análisis del carbono orgánico a $1100{ }^{\circ} \mathrm{C}$ en presencia de carbonato de calcio haciendo el análisis en 2 minutos para evitar la descomposición de los carbonatos.

Chichester y Chaison (1992) (12), realizaron la determinación de carbono orgánico a $575{ }^{\circ} \mathrm{C}$ en muestras de suelos con un contenido de carbono orgánico entre $1,00-4,00 \%$, y la determinación de carbono inorgánico a $1000{ }^{\circ} \mathrm{C}$. Para validar los resultados de $\mathrm{C}$ orgánico obtenidos, realizaron un tratamiento previo con ácido a las muestras, y midieron el carbono a $1300{ }^{\circ} \mathrm{C}$ de las muestras tratadas. Los resultados obtenidos por ambos métodos fueron coincidentes, pero los valores de carbono orgánico son muy altos si se comparan con los que se pretenden medir en este trabajo.

En resumen, se puede decir que existe una gran diversidad de métodos para llevar a cabo la determinación de carbono orgánico con conclusiones muy diferentes, y en ninguno de ellos se aborda el análisis de carbono orgánico en presencia de siderita, por lo que, de acuerdo a la bibliografía consultada y a la información disponible en nuestro laboratorio por estudios previos, se decidió trabajar a cuatro temperaturas: $450{ }^{\circ} \mathrm{C}$, $490{ }^{\circ} \mathrm{C}, 520^{\circ} \mathrm{C}$ y $540{ }^{\circ} \mathrm{C}$ para determinar la temperatura a la cual se consigue una recuperación del analito del $100 \%$. La muestra ensayada fue la ARCILLA T, cuya concentración de C orgánico era conocida, y a la cual se había adicionado un $5 \%$ de siderita para determinar a qué temperatura se conseguía una interferencia mínima de la siderita. La determinación se llevó a cabo con el analizador RC-412 manteniendo la muestra durante 400 segundos a la temperatura máxima.

Paraelestudiodelainterferencia delasideritaenla determinación de carbono orgánico, se prepararon mezclas con las arcillas 1 a 6, a las que se adicionaron diferentes cantidades de siderita $(3 \%$ y $5 \%$ de siderita). Para la preparación de dichas mezclas se pesó la cantidad adecuada de los materiales, y se molturaron en un molino de anillos de carburo de wolframio para conseguir muestras totalmente homogéneas y con un tamaño de partícula inferior a 75 $\mu$ m que es el adecuado para llevar a cabo la determinación.

Los valores obtenidos se compararon con los recomendados en los certificados de cada uno de ellos. La comparación de las medidas de los materiales de referencia con los valores de referencia que aparecen en los certificados se ha realizado aplicando el test de Sutarno-Steger para determinar la exactitud del método. Dicho test establece que el valor medio obtenido puede ser aceptado si se cumple:

$$
z=\frac{\left|\mathbf{x}_{\text {obtenido }}-\mathbf{x}_{\text {conocido }}\right|}{\mathbf{s}_{\text {conocido }}}<2
$$

donde $\mathbf{x}_{\text {obtenido }}$ es el valor medio obtenido del elemento analizado, $\mathbf{x}_{\text {conocido }}$ es el valor certificado o recomendado y $\mathbf{s}_{\text {conocido }}$ es el valor de la desviación estándar o incertidumbre del valor certificado o recomendado (26).

\subsubsection{ELECCIÓN DEL EQUIPO MÁS ADECUADO PARA LA DETERMINACIÓN DE C ORGÁNICO}

Con objeto de determinar cuál de los equipos disponibles en el Laboratorio de Análisis Químico del ITC es más adecuado para llevar a cabo la determinación de carbono orgánico se analizaron los materiales de referencia mediante el analizador RC-412 y el TruSpec CHN a una temperatura de $490{ }^{\circ} \mathrm{C}$. Los resultados se compararon usando el test de Sutarno-Steger definido en el apartado 2.3.1.

TAbla II. Programas utilizados PARA LleVAR A CABO LAS MEDidAS MEDIANTE EL RC-412 Y MEDIANTE EL TRUSPEC CHN

\begin{tabular}{|c|c|c|c|}
\hline \multicolumn{2}{|c|}{ RC-412 } & \multicolumn{2}{c|}{ TruSpec CHN } \\
\cline { 1 - 2 } $\begin{array}{c}\text { Temperatura } \\
\left({ }^{\circ} \mathbf{C}\right)\end{array}$ & $\begin{array}{c}\text { Tiempo } \\
(\text { segundos) }\end{array}$ & $\begin{array}{c}\text { Temperatura } \\
\left({ }^{\circ} \mathbf{C}\right)\end{array}$ & $\begin{array}{c}\text { Tiempo } \\
\text { (segundos) }\end{array}$ \\
\hline $25-490$ & 100 & \multirow{2}{*}{490 (T máx) } & 400 \\
\hline 490 (T máx) & 400 & & \\
\hline
\end{tabular}

En la tabla II se detallan los programas de calentamiento (temperatura y tiempo de permanencia) utilizados para llevar a cabo las medidas con los dos equipos utilizados. 


\subsubsection{DETERMINACIÓN DE C ORGÁNICO EN} UN EQUIPO CON HORNO DE INDUCCIÓN CON TRATAMIENTO PREVIO DE LAS MUESTRAS

Cuando no se dispone de un equipo capaz de trabajar a temperaturas variables (horno eléctrico), sino de uno con horno de inducción, para llevar a cabo el análisis del carbono orgánico es necesario realizar un tratamiento previo de las muestras. Dicho tratamiento debe eliminar las interferencias que pudiera provocar el C inorgánico procedente de la siderita, calcita, dolomita, etc. en la medida del C orgánico. Para ello, las muestras se sometieron a un tratamiento previo con $\mathrm{HCl}$ al $50 \%$.

Para el tratamiento previo de las muestras con $\mathrm{HCl}$, se tomaron 5,0000 g de muestra a los que se añadieron $30 \mathrm{~mL}$ de $\mathrm{HCl} 50 \%$. La mezcla se agitó durante aproximadamente 30 minutos, hasta que terminó de burbujear y a continuación se filtró. El sólido obtenido se lavó con agua destilada y se secó a $110^{\circ} \mathrm{C}$.

Las muestras ensayadas fueron las Arcillas 1 a 6 sin adición de siderita y con una adición de un $5 \%$ de siderita. Se llevó a cabo la determinación de $\mathrm{C}$ orgánico a $490{ }^{\circ} \mathrm{C}$ en el equipo con horno eléctrico (RC-412) y también se realizó la determinación de carbono de las muestras tratadas previamente con $\mathrm{HCl}$ en el equipo con horno de inducción (CS-200).

Para la comparación de los resultados se utilizó el test de Sutarno-Steger definido en el apartado 2.3.1.

\subsubsection{ESTUDIO DE LA RELACIÓN ENTRE LA CONCENTRACIÓN DE CARBONO ORGÁNICO Y LA FORMACIÓN DE CORAZÓN NEGRO}

Para el estudio de la relación entre la concentración de carbono orgánico presente en los materiales y la formación de corazón negro en las piezas preparadas con dichos materiales durante el proceso de cocción, se llevó a cabo la determinación de carbono orgánico a $490{ }^{\circ} \mathrm{C}$ con el analizador RC-412 en tres arcillas y tres atomizados. Los materiales utilizados para este estudio fueron las Arcillas A, B y C, y los Atomizados A, B y C. Además, se realizó un estudio de la tendencia a la formación de corazón negro en las piezas preparadas con dichos materiales.

La tendencia a la formación de corazón negro, se determinó sobre probetas de cada una de las muestras, conformadas a una humedad del 5,5\% en peso y a una presión de prensado de $300 \mathrm{~kg} \cdot \mathrm{cm}^{-2}$ en el caso de las arcillas y de $400 \mathrm{~kg} \cdot \mathrm{cm}^{-2}$ en los atomizados. Dichas probetas se cocieron en un horno eléctrico de laboratorio a una temperatura máxima de $1120^{\circ} \mathrm{C}$ para las arcillas y de $1200^{\circ} \mathrm{C}$ para los atomizados, con un tiempo de permanencia a esta temperatura de 1 minuto y una velocidad de calentamiento de $25^{\circ} \mathrm{C} / \mathrm{min}$. Posteriormente se cortó una sección transversal y se observó la extensión de la zona oxidada.

\section{RESULTADOS}

3.1. Optimización de la temperatura de trabajo para la medida directa de $\mathrm{C}$ orgánico en un horno eléctrico y efecto de la presencia de siderita

En la tabla III se detallan los resultados obtenidos en la determinación de $\mathrm{C}$ orgánico a las cuatro temperaturas estudiadas en la ARCILLA T con una adición de siderita del $5 \%$ junto con su incertidumbre. Los valores que se muestran son la media aritmética de 10 determinaciones.

La incertidumbre se ha calculado con la expresión:

$$
U=t \cdot \frac{s}{\sqrt{n}}
$$

Donde $U$ es la incertidumbre de la medida para un intervalo de confianza del $95 \%$, s es la desviación estándar de las medidas, $t$ es el valor estadístico de $t$ de Student para un intervalo de confianza del $95 \%$ y $n$ es el número de medidas de la muestra bajo condiciones de reproducibilidad (27).

La temperatura de trabajo óptima obtenida fue de $490{ }^{\circ} \mathrm{C}$, pues trabajando a la temperatura de $450{ }^{\circ} \mathrm{C}$, no se obtenía una recuperación del analito del $100 \%$, y a las temperaturas de $520^{\circ} \mathrm{C}$ y $540{ }^{\circ} \mathrm{C}$ se observaron valores más elevados lo que indicaba que posiblemente se había producido la descomposición de parte de la siderita. Los valores de $\mathrm{z}$ calculados para comparar los resultados indican que la temperatura a la que se obtienen resultados que no son significativamente diferentes del valor conocido es la de $490{ }^{\circ} \mathrm{C}$, que es la que presenta un valor de $\mathrm{z}$ inferior. El

TABlA III: Resultados de LA DETERMINACIÓN DE C ORGÁNICO EN la ARCILLA T CON UN 5 \% DE SIDERITA MEDIANTE EL ANALIZADOR RC-412 A LAS TRES TEMPERATURAS ESTUDiADAS $450{ }^{\circ} \mathrm{C}, 490{ }^{\circ} \mathrm{C}, 520^{\circ} \mathrm{C}$ Y $540{ }^{\circ} \mathrm{C}$

\begin{tabular}{|c|c|c|c|c|c|c|c|c|c|c|c|c|c|}
\hline \multirow{2}{*}{ Muestra } & \multirow{2}{*}{$\% \mathrm{C}_{\text {org conocido }}$} & \multicolumn{3}{|c|}{$\mathrm{T}=450^{\circ} \mathrm{C}$} & \multicolumn{3}{|c|}{$\mathrm{T}=490^{\circ} \mathrm{C}$} & \multicolumn{3}{|c|}{$\mathrm{T}=520^{\circ} \mathrm{C}$} & \multicolumn{3}{|c|}{$\mathrm{T}=540^{\circ} \mathrm{C}$} \\
\hline & & $\% \mathrm{C}_{\text {org }}$ & $u$ & $z$ & $\% \mathrm{C}_{\mathrm{org}}$ & $u$ & $z$ & $\% \mathrm{C}_{\text {org }}$ & $U$ & $z$ & $\% \mathrm{C}_{\text {org }}$ & $u$ & $z$ \\
\hline Arcilla T & $0,037 \pm 0,002$ & 0,032 & 0,005 & 2,5 & 0,037 & 0,005 & 0 & 0,042 & 0,005 & 1,0 & 0,047 & 0,007 & 5,0 \\
\hline
\end{tabular}

TABla IV: RESUltAdOS DE LA DETERMINACIÓN DE C ORGÁNICO EN LAS ARCILLAS CON DIFERENTES ADICIONES DEL PATRÓN NCS DC 14038 SidERITE MEDIANTE EL ANALIZADOR RC-412 A $490^{\circ} \mathrm{C}$

\begin{tabular}{|c|c|c|c|c|c|}
\hline \multirow{2}{*}{ Muestra } & \multirow{2}{*}{$\begin{array}{c}\left(\% \mathrm{C}_{\text {org }}\right) \text { ARCILLA (sin } \\
\text { adición) }\end{array}$} & \multicolumn{4}{|c|}{$\left(\% \mathrm{C}_{\text {org }}\right)$ ARCILLA (con adición) } \\
\hline & & $3 \%$ NCS Siderite & $z$ & $5 \%$ NCS Siderite & $z$ \\
\hline Arcilla1 & $0,35 \pm 0,02$ & $0,34 \pm 0,02$ & 0,50 & $0,35 \pm 0,02$ & 0,00 \\
\hline Arcilla 2 & $0,09 \pm 0,01$ & $0,08 \pm 0,01$ & 1,00 & $0,09 \pm 0,01$ & 0,00 \\
\hline Arcilla 3 & $0,11 \pm 0,01$ & $0,11 \pm 0,01$ & 0,00 & $0,12 \pm 0,01$ & 1,00 \\
\hline Arcilla 4 & $0,21 \pm 0,02$ & $0,22 \pm 0,02$ & 0,50 & $0,22 \pm 0,02$ & 0,50 \\
\hline Arcilla 5 & $0,60 \pm 0,03$ & $0,59 \pm 0,03$ & 0,33 & $0,58 \pm 0,02$ & 0,66 \\
\hline Arcilla 6 & $0,77 \pm 0,04$ & $0,76 \pm 0,03$ & 0,25 & $0,78 \pm 0,03$ & 0,25 \\
\hline
\end{tabular}


resultado difiere de lo que se muestra en la bibliografía, ya que en el trabajo de Chichester y Chaison (12) se trabaja a una temperatura de $575{ }^{\circ} \mathrm{C}$ para la determinación de $\mathrm{C}$ orgánico y se obtienen buenos resultados. Esto podría ser debido a que como el material analizado en dicho estudio no presentaba siderita en su composición no se observó la interferencia del C inorgánico en la medida del C orgánico.

En la tabla IV se muestran los resultados obtenidos en la determinación de carbono orgánico con el analizador RC-412 a $490{ }^{\circ} \mathrm{C}$, en las muestras de arcilla sin adición y con adición de diferentes cantidades del material NCS DC 14038 Siderite (NCS Siderite), junto con su incertidumbre.

Los resultados obtenidos muestran valores de $\mathrm{z}$ que indican que no se observan diferencias significativas entre los valores de $\mathrm{C}$ orgánico de las muestras sin adición de siderita y con adición de siderita, lo cual confirma que a la temperatura de trabajo $\left(490^{\circ} \mathrm{C}\right)$, y con el equipo utilizado, no se produce una interferencia apreciable de la siderita en la determinación de $\mathrm{C}$ orgánico a las concentraciones ensayadas.

\subsection{Elección del equipo más adecuado para la determinación de C orgánico}

En la tabla V se detallan los resultados obtenidos en la determinación de carbono orgánico en los materiales de referencia utilizando los dos analizadores elementales: RC-412 y TruSpec $\mathrm{CHN}$ a una temperatura de $490^{\circ} \mathrm{C}$.

Se detalla la media de los resultados de carbono orgánico obtenidos en la medida de cada uno de los materiales de referencia, junto con su incertidumbre (u), el número de datos obtenidos en el laboratorio (n), y el valor de $z$ obtenido en la comparación de los resultados obtenidos con el valor recomendado en el certificado (tal y como se explica en el apartado 2.3.1.).

A la vista de los resultados obtenidos se puede decir que ambos equipos son adecuados para la medida ya que con los dos se obtienen buenos resultados, con valores de $\mathrm{z}$ inferiores a 2. Aunque cabe destacar que con el analizador TruSpec CHN se obtienen valores de incertidumbre más pequeños.

\subsection{Determinación de $\mathrm{C}$ orgánico en un equipo con horno de inducción con tratamiento previo de las muestras}

En la tabla VI se muestran los resultados obtenidos en la determinación de $\mathrm{C}$ orgánico en el equipo con horno de eléctrico (RC-412 a $490{ }^{\circ} \mathrm{C}$ ) y con horno de inducción (CS-200) de las arcillas con y sin adición de siderita tras el tratamiento con $\mathrm{HCl}$.

Para comparar los resultados obtenidos se utilizó el test de Sutarno-Steger tal y como se describe en el apartado 2.3.1. Se puede observar que los resultados de la determinación de carbono orgánico mediante un equipo con horno de inducción, después de someter la muestra a un tratamiento adecuado con $\mathrm{HCl}$, coinciden con los valores de carbono orgánico hallados mediante la medida con un horno eléctrico a $490{ }^{\circ} \mathrm{C}$, ya que el valor obtenido de $\mathrm{z}$ siempre es inferior a 2 .

Por lo tanto, se puede decir que es posible determinar el carbono orgánico con un equipo con horno de inducción tratando la muestra con una disolución de $\mathrm{HCl}$ al $50 \%$ para eliminar los carbonatos, aunque el límite de detección en la determinación de carbono orgánico sería superior al de un equipo capaz de trabajar a temperatura programable.

TABla V: Resultados DE LA DETERMINACIÓN DE CARBONO ORGÁNICO EN UNA SERIE DE MATERIALES DE REFERENCIA DE SUELOS MEDIANTE EL ANALIZADOR RC-412 Y EL ANALIZADOR TRUSPEC

\begin{tabular}{|c|c|c|c|c|c|c|c|c|c|}
\hline \multirow{2}{*}{ Material de referencia } & \multirow{2}{*}{$\begin{array}{c}\text { Valor conocido } \\
\left(\% \mathrm{C}_{\mathrm{org}}\right)\end{array}$} & \multicolumn{4}{|c|}{$\mathrm{RC}-412\left(490^{\circ} \mathrm{C}\right)$} & \multicolumn{4}{|c|}{ TruSpec CHN $\left(490^{\circ} \mathrm{C}\right)$} \\
\hline & & $\mathrm{X}_{\text {obtenido }}$ & $\mathbf{u}(\%)$ & $\mathbf{n}$ & $\mathbf{z}$ & $\begin{array}{l}\mathrm{X}_{\text {obtenido }} \\
\left(\% \mathrm{C}_{\text {org }}\right)\end{array}$ & $\mathbf{u}(\%)$ & $\mathrm{n}$ & $\mathbf{z}$ \\
\hline GBW 07401 & $1,80 \pm 0,16$ & 1,78 & 0,04 & 10 & 0,12 & 1,81 & 0,03 & 10 & 0,06 \\
\hline GBW 07402 & $0,49 \pm 0,07$ & 0,50 & 0,02 & 10 & 0,14 & 0,51 & 0,01 & 10 & 0,29 \\
\hline GBW 07403 & $0,51 \pm 0,03$ & 0,51 & 0,02 & 10 & 0,00 & 0,52 & 0,01 & 10 & 0,33 \\
\hline GBW 07406 & $0,81 \pm 0,09$ & 0,79 & 0,03 & 10 & 0,22 & 0,82 & 0,02 & 10 & 0,11 \\
\hline GBW 07407 & $0,64 \pm 0,07$ & 0,63 & 0,03 & 10 & 0,14 & 0,66 & 0,02 & 10 & 0,29 \\
\hline
\end{tabular}

TABla VI: Resultados DE LA DETERMINACIÓN DE C ORGÁNICO CON EL EQUIPO RC-412 A 490 C Y DE C TOTAL CON EL EQUIPO CS-200 TRAS EL TRATAMIENTO DE LA MUESTRA CON HCL

\begin{tabular}{|c|c|c|c|c|c|c|c|}
\hline \multirow{2}{*}{ Muestra } & \multicolumn{3}{|c|}{$\begin{array}{c}\% \mathrm{C}_{\mathrm{org}} \\
\left.\text { (Horno eléctrico a } 490{ }^{\circ} \mathrm{C}-\mathrm{RC}-412\right)\end{array}$} & \multicolumn{4}{|c|}{$\begin{array}{c}\% \mathrm{C}_{\text {total }} \\
\text { (Horno de inducción - CS-200) Muestra tratada con } \mathrm{HCl}\end{array}$} \\
\hline & $\begin{array}{l}\text { Sin adición de } \\
\text { siderita }\end{array}$ & $\begin{array}{l}\text { Con } 5 \% \text { NCS } \\
\text { Siderite }\end{array}$ & $\mathbf{z}$ & $\begin{array}{l}\text { Sin adición de } \\
\text { siderita }\end{array}$ & $\mathbf{z}$ & $\begin{array}{l}\text { Con } 5 \% \text { NCS } \\
\text { Siderite }\end{array}$ & $\mathbf{z}$ \\
\hline Arcilla 1 & $0,35 \pm 0,02$ & $0,34 \pm 0,03$ & 0,50 & $0,36 \pm 0,03$ & 0,50 & $0,36 \pm 0,03$ & 0,50 \\
\hline Arcilla 3 & $0,11 \pm 0,01$ & $0,12 \pm 0,01$ & 1,00 & $0,11 \pm 0,01$ & 0,00 & $0,12 \pm 0,01$ & 1,00 \\
\hline Arcilla 4 & $0,21 \pm 0,02$ & $0,21 \pm 0,01$ & 0,00 & $0,21 \pm 0,03$ & 0,00 & $0,20 \pm 0,02$ & 0,50 \\
\hline Arcilla 5 & $0,60 \pm 0,03$ & $0,61 \pm 0,03$ & 0,33 & $0,59 \pm 0,04$ & 0,33 & $0,58 \pm 0,03$ & 0,66 \\
\hline Arcilla 6 & $0,77 \pm 0,04$ & $0,78 \pm 0,03$ & 0,25 & $0,78 \pm 0,05$ & 0,25 & $0,79 \pm 0,05$ & 0,50 \\
\hline
\end{tabular}


TABLA VII: RESULTADOS OBTENIDOS EN LA DETERMINACIÓN DE C ORGÁNICO EN ARCILLAS Y ATOMIZADOS Y FOTOGRAFÍAS DE LA FORMACIÓN DE CORAZÓN NEGRO TRAS EL PROCESO DE COCCIÓN

\begin{tabular}{|c|c|c|}
\hline Muestra & $\begin{array}{c}\% \text { C orgánico } \\
\left(\mathbf{4 9 0}{ }^{\circ} \mathrm{C}\right)\end{array}$ & $\begin{array}{c}\text { Fotografías de las } \\
\text { probetas tras el ensayo }\end{array}$ \\
\hline Arcilla A & $0,32 \pm 0,02$ & \\
\hline Arcilla B & $0,15 \pm 0,01$ & \\
\hline Arcilla C & $0,03 \pm 0,01$ & \\
& & \\
\hline
\end{tabular}

\subsection{Estudio de la relación entre la concentración de carbono orgánico y la formación de corazón negro}

En la tabla VII se detallan los resultados obtenidos en la determinación de carbono orgánico de las muestras sobre las que se han llevado a cabo los ensayos de formación de corazón negro, así como, las fotografías correspondientes a una sección de las probetas obtenidas tras el ensayo de tendencia a la formación del corazón negro.

De acuerdo a los resultados obtenidos, se puede decir que las muestras en las que se aprecia claramente la formación de corazón negro son las arcillas A y B, que son aquéllas que presentan una concentración mayor de $\mathrm{C}$ orgánico.

Además, existe una buena correlación entre los valores de carbono orgánico y la tendencia a la formación de corazón negro (en las condiciones en las que se ha llevado a cabo el ensayo) aunque sólo ha sido posible observar la presencia de corazón negro en valores de carbono superiores a 0,08 \%.

Por todo ello, se puede decir que el método de determinación de carbono orgánico es mucho más sensible a la presencia de materia orgánica que el ensayo de tendencia a la formación de corazón negro (apartado 2.3.4). Sin embargo, es el ensayo de tendencia a la formación de corazón negro el que reproduce las condiciones industriales de uso de los materiales y, por ello, proporciona una valiosa información acerca del problema que puede generar la materia orgánica en un material, mientras que el valor de carbono orgánico, sin otra información, no sería suficiente para poder predecir el comportamiento de los materiales, ya que las condiciones de procesado del material influyen de forma muy importante sobre la tendencia a la formación de corazón negro.

\section{CONCLUSIONES}

Del estudio realizado se pueden extraer las siguientes conclusiones:

1) La temperatura de trabajo más adecuada para llevar a cabo la determinación de C orgánico, obteniendo una recuperación del analito del $100 \%$ y sin que se observe una interferencia de la siderita presente en los materiales, es de $490^{\circ} \mathrm{C}$.

\begin{tabular}{|c|c|c|}
\hline Muestra & $\begin{array}{c}\% \text { C orgánico } \\
\left(\mathbf{4 9 0}{ }^{\circ} \mathbf{C}\right)\end{array}$ & $\begin{array}{c}\text { Fotografías de las } \\
\text { probetas tras el ensayo }\end{array}$ \\
\hline Atomizado A & $0,01 \pm 0,01$ & \\
\hline Atomizado B & $0,05 \pm 0,01$ & \\
\hline Atomizado C & $0,08 \pm 0,01$ & \\
& & \\
\hline
\end{tabular}

2) El equipo óptimo para llevar a cabo el análisis de C orgánico sin necesidad de un tratamiento previo de la muestra es el analizador elemental TruSpec CHN por presentar incertidumbres inferiores, pero también se obtienen buenos resultados con el analizador RC-412, ya que las incertidumbres que se obtienen también son bajas y permiten obtener resultados precisos y exactos.

3) La determinación de C orgánico se puede llevar a cabo con un equipo que disponga de un horno de inducción, si se realiza un tratamiento previo de la muestra con una disolución de $\mathrm{HCl}$, y se eliminan completamente los carbonatos presentes en el material a analizar.

4) Existe una correlación entre la concentración de carbono orgánico y la aparición de corazón negro, y se ha observado que el método de determinación de $\mathrm{C}$ orgánico es más sensible a la presencia de materia orgánica que el ensayo de formación de corazón negro. Sin embargo, únicamente el valor de $\mathrm{C}$ orgánico, no sería suficiente para predecir el comportamiento del material, ya que las condiciones de procesado del material influyen de forma muy importante sobre la tendencia a la formación de corazón negro.

\section{AGRADECIMIENTOS}

Este trabajo ha sido financiado por el Instituto de la Mediana y Pequeña Industria Valenciana dentro del programa PRODEIT Programa de desarrollo estratégico en el Plan de ayuda a Institutos Tecnológicos, a través del proyecto IMDEEA/2011/82, a través del Fondo Europeo de Desarrollo Regional de la Unión Europea.

\section{BIBLIOGRAFÍA}

1. M.F. GAZULLA, M.J. ORTS, A. GOZALBO, J.L. AMORÓS. Determinación del contenido en materia orgánica en arcillas por culombimetría. XVII REUNION CIENTIFICA DE LA SOCIEDAD ESPAÑOLA DE ARCILLAS (SEA 2002) Elche y Castellón 27 - 30 Noviembre, 2002 Publicado en: Sanfeliu, T.; Jordán, M.M. (Eds.) La investigación de arcillas en geología, agricultura, medio ambiente y ciencia de materiales. Elche: Universidad Miguel Hernández, 2002. pp. 229-232.

2. A. BARBA; A. MORENO; F. NEGRE; A. BLASCO. Oxidation of black cores in firing. Tile Brick Int., 6(5), 17-23, 1990. 
3. A. ESCARDINO; A. BARBA; A. BLASCO; F. NEGRE. Oxidation of black core during firing of ceramic ware: 4 . Relations ship between effective diffusivity of oxygen through oxidised layer and properties characterising its porous structure. Br. Ceram. Trans., 94(3), 103-108, 1995.

4. A. BARBA JUAN. Oxidación del "corazón negro" durante la cocción de piezas cerámicas. Influencia de la estructura del sólido y de su contenido en materia orgánica y óxidos de hierro sobre la cinética del proceso. Valencia: Universidad de Valencia, 1989. Tesis doctoral.

5. E. PERALTA REYES. Estudio de los óxidos de hierro en la cerámica negra y de corazón negro. Universidad Autonoma Metropolitana Iztapalapa (Mejico), 2004 . Trabajo para obtener el grado de Maestría en Ingenieria Química.

6. B.M. FRENCH, P.E. ROSENBERG. Siderite $\left(\mathrm{FeCO}_{3}\right)$ : thermal decomposition in equilibrium with graphite. Science 147 (3663), 1283-1284, 1965.

7. H.E. KISSINGER, H.F. McMURDIE, B.S.SIMPSON. Thermal decomposition of manganous and ferrous carbonates. J. Am. Ceram. Soc., 39(5), 168-172, 1956.

8. R.A. ROWLAND, E.C. JONAS. Variations in differential thermal analysis curves of siderite. American Mineralogist, 34(7-8), 550-558, 1947.

9. F.L. CUTHBERT, R.A. ROWLAND. Differential thermal analysis of some carbonate minerals. American Mineralogist ,32(3-4),111-116, 1947.

10. N.V. SIDGWICK. The chemical elements and their compounds, Vol, II, Oxford University Press, London, 1950.

11. L.E. ALLISON. Wet-combustion apparatus and procedure for organic and inorganic carbon in soil. Soil science society proceedings 24, 36-40, 1960

12. F.W. CHICHESTER, R.F. CHAISON. Analysis of carbon in calcareous soils using a two temperature dry combustion infrared instrumental procedure. Soil Science 153 (3), 237-241, 1992.

13. UNE EN 13137: 2002 (Comité: 77) Caracterización de residuos. Determinación de carbono orgánico total (TOC) en residuos, lodos y sedimentos

14. Methods of Soil Analysis. Part 3. Chemical Methods. XX ed. USA: Editorial, 1996. Chap. 34, Total Carbon, Organic Carbon and Organic Matter, pp. 9611010.

15. ASTM D 7573: 2009. Standard test method for total carbon and organic carbon in water by high temperature catalytic combustion and infrared detection.
16. G.L. KERVEN, N.W. MENZIES, M.D. GEYER. Soil carbon determination by high temperature combustion - a comparison with dichromate oxidation procedures and the influence of charcoal and carbonate carbon on the measured value. Communications in soil science and plant analysis 31, 19351939, 2000.

17. C. CIAVATTA, L.V. ANTISARI, P. SEQUI. Determination of organic carbon in soils and fertilizers. Communications in soil science and plant analysis 20, 759-773, 1989 .

18. C.J. SCHOLLENBERGER. Determination of soil organic matter. Soil science 59, 53-56, 1945

19. M. RAIMONDO, P. DANASINO, M. DONDI. Determinazione quantitativa del carbonio organico nei materiali argillosi per uso ceramico: un confronto fra tre diversi metodi analitici. Ceramurgia 29(3), 179-186, 1999

20. MURRAY, H.H. Developments in Clay Science. Volumen 2: Applied Clay Mineralogy. Elsevier B.V. 2006. Appendix B: Common Tests for Evaluation of ball Clay Samples, p. 161-168.

21. I. MATEJOVIC. Determination of carbon and nitrogen in samples of various soils by the dry combustion. Commun. Soil Sci. Anal. 28, 1499-1511, 1997.

22. M.F. GAZUlla, M. RODRIGO, M. ORDUÑA, C.M. GÓMEZ Determination of carbon, hydrogen, nitrogen and sulfur in geological materials using elemental analysers. Geostand. Geoanal. Res., DOI: 10.1111/ j.1751-908X.2011.00140.x

23. LARSON, E.T., HEIKOOP, J.M., PERKINS, G.; CHIPERA, S.J.; HESS, M.A Pretreatment technique for siderite removal for organic carbon isotope and C:N ratio analysis in geological samples. Rapid Commun Mass Spectrom., 22(6), 865-72, 2008.

24. M.D. KROM, R.A. BERNER. A rapid method for the determination of organic and carbonate carbon in geological samples. Journal of sedimentary petrology 53, 660-663, 1983

25. D.WANG, D.W. ANDERSON. Direct measurement of organic carbon content in soils by the LECO CR-12 Carbon analyzer. Commun. Soil Sci. Anal., 29(1\&2), 15-21, 1998.

26. R. SUTARNO, H.F. STEGER. Validation of accuracy by interlaboratory programme. Talanta, 32, 1088-1091, 1985.

27. Use of Reference Materials and the Estimation of Measurement Uncertainty. Course Material. Joint Research Center. Institute for Reference Materials and Measurements (15-16 October 2008). 